\title{
Slip-sliding away
}

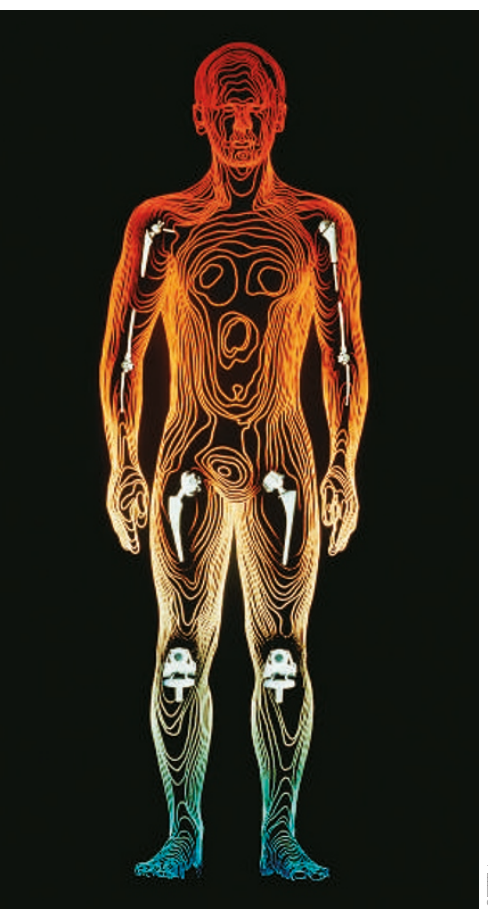

Wear and tear in biomedical implants and engineering devices are often reduced by boundary lubrication, in which the sliding surfaces are coated with a monomolecular layer of surfactants that creates a slip plane between the two surfaces. A team of researchers now proposes that in water, the slip plane is at the surfactant-surface interface, rather than the surfactant-surfactant interface as in air (W. H. Briscoe et al. Nature 444, 191-194; 2006). Jacob Klein and co-workers used a surface force apparatus to measure the normal and shearing forces between two mica surfaces covered by a cationic surfactant having a double alkyl chain. The cationic headgroups of the surfactant attach to the mica surfaces, leaving the alkyl chains extending into the space between the two surfaces. In water the forces were reduced by $99 \%$ compared with those in air. The authors suggest this is due to the hydration of the headgroups, swelling that region and facilitating their lateral motion on the surface - this then becomes the interface that is least resistant to shear. These findings could aid more specific design of boundary lubricant molecules for use in devices that can deteriorate due to friction.

\section{SURFACE PLASMONS JUMP THROUGH HOOPS}

Surface plasmon polaritons (SPPs) — electromagnetic waves at the surface of a metal — have seen a significant surge of interest based on their ability to localize optical fields at length scales much smaller than their wavelength. However, their use is limited to wavelengths near the plasmon frequency of the metal, which typically lies in the ultraviolet region and it has been difficult to extend the use of SPPs to other wavelengths. In particular, there is significant interest to use this technology at long wavelengths such as terahertz, where guiding light is a difficult task. Now, Stefan Maier and colleagues propose an ingenious technique to extend surface plasmon technology to almost arbitrary wavelengths (Phys. Rev. Lett. 97, 176805; 2006). In their approach, a cylindrical wire is periodically structured with radial grooves. The periodic structures, matched to the desired wavelength, lead to localization of the optical field at the surface of the wire. In their calculations, Maier and colleagues show that these structures can be used for waveguiding as well as focusing.

\section{Shrinking nanotubes}

The physical properties of carbon nanotubes strongly depend on their dimensions with obvious technological consequences for a wide variety of nanoscale devices including transistors, actuators and sensors. However, current preparation methods cannot reliably control nanotube diameter, thereby preventing the predictable fabrication process of these devices. Alex Zettl and colleagues now report on a way to controllably alter the diameter of an individual carbon nanotube (Nano Lett. doi:10.1021/nl061671j; 2006). This is achieved by combining defect formation via electron irradiation with simultaneous electromigration in vacuum causing the formation of highquality nanotubes of successively smaller diameter. The formation process observed by transmission electron microscopy can be stopped at any diameter down to $1 \mathrm{~nm}$, below which the inner wall of the tube finally breaks. Electronic transport measurements show clear dependence of conductance on nanotube geometry. The authors believe that such characteristics could serve as templates for single molecule electronics.

\section{Microgels with a twist}

Researchers in the US have succeeded in combining light, heat and pH responsiveness in a poly $(N$-isopropylacrylamide $)$ microgel, by linking a photochromic moiety called spiropyran to its amide groups

\section{(A. Garcia et al. Langmuir} doi:10.1021/la061632n; 2006). Spiropyrans are clever little molecules. Their chemical structure contains two rings joined by a shared carbon atom. whose bonds are therefore rather strained. On irradiation with ultraviolet light, one of these bonds snaps, opening up one ring so that the whole molecule becomes more extended. By cycling between dark ultraviolet and visible irradiation, the process is made reversible, and the authors have exploited this in order to change the volume of the microgel particles. These particles are made from a copolymer that is thermosensitive so the volume can also be adjusted by heating or cooling the material In the extended form, the molecule is hydrophilic and can become positively charged if the $\mathrm{pH}$ is lowered. The authors suggest that this can be used to control the hydration of microgels. Moreover, the copolymer itself has pH-sensitive moieties that cause the particles to shrink or swell at high or low pH values, respectively.

\section{Tiny hand with tight grip}

Yen-Wen Lu and Chang-Jin Kim from the University of California in Los Angeles have built a micromechanical device on a submillimetre scale that imitates the functions of a hand (Appl. Phys. Lett. 89, $16410 ; 2006$ ). The four minifingers are fabricated by lithographically defining six phalanges on a silicon wafer. Polymer inflatable balloons deposited on the phalanges pose as the muscles: when they are inflated they shorten the distance between the phalanges, resulting in flexion of the fingers, as shown in the figure (deflation results in extension). Besides reproducing the basic motion of a human hand, the researchers demonstrated the possibility of grabbing and manipulating biological systems of submillimetre dimensions. This is not the first time that a microelectromechanical device with complex functions has been developed. However, the fabrication from a single block of material makes this one particularly strong. In addition, because the microhand is inert to the surroundings, it can operate in air or water environments, making it a promising tool for biological applications. 